\title{
Virtual Factory Framework: An Innovative Approach to Support the Planning and Optimization of the Next Generation Factories
}

\author{
A. Azevedo*. R. P. Francisco*. \\ J. Bastos*. A. Almeida* \\ *Inesc Porto and Faculdade de Engenharia da Universidade do Porto, Rua Dr. Roberto Frias S/N \\ 4200-465 Porto, Portugal (e-mail: ala, roberto.piedade, joao.bastos,ee05019@fe.up.pt).
}

\begin{abstract}
Industrial managers are in the expectation that the new generation factories bring more competitiveness to the manufacturing companies through innovative approaches, methods and tools that support the factory planning process. The concept of "factory as a product" perceives the factory as a complex long life product introducing design advantages for factory planning and optimization, seeking to improve quality, reduce ramp-up, product delivery and overall reduce time and costs. In order to attain these objectives, there is an urgent need for an innovative and suitable Virtual Factory Framework to structure the development of new generation factories.
\end{abstract}

\section{INTRODUCTION}

Current approaches supporting planning and optimization of factories have shown limitations and an inability to address the increasing market competiveness and responsiveness. There is an urgent need for new approaches that address these current market challenges. This work presents a new approach currently being developed by a research consortium involving academic partners, research institutes, manufacturing and software development companies.

It is agreed that modern factories must be open, agile, flexible, modular, scalable, and knowledge-based in order to adapt, in real time, to the changes caused by new market demands, technology advances and sustainability issues, among other concerns. In a manufacturing context, these challenges impose the design of future factories that could improve knowledge and performance as far as the design, management, evaluation and redesign of new or existing factories are concerned.

This improvement can be supported through the ability to simulate the complex dynamic behavior throughout the factory life-cycle, synchronizing the Virtual Factory with the Real Factory. The process planning can be carried out through basic components that can be simulated by aligning the design of the factory and decision-making process during the factory life-cycle.

This work intends to present the Virtual Factory Framework, which is an innovative approach to plan and optimize the factory design. Chapter two presents the concept of "factory as a product"; chapter three addresses the life-cycle paradigm to describe planning and optimization. Then, the VFF Project is presented, as well the main outcomes in this work, the factory templates. Finally, some conclusions are presented, including further works expected as a consequence of this ongoing research project.

\section{CONCEPT OF "FACTORY AS A PRODUCT"}

In the factory development project, the factory can be understood as a special product that requires a complex lifecycle using principles and methods currently developed and used for product development. The new concept of "factory as a product" may be introduced to support the manufacturing companies in the search for new solutions (products, processes and technology) in order to improve quality, reduce time to market, to achieve more efficient ramp-up to processes and factories, on time product delivery and also reduced production costs, thus leading to a greater competitiveness.

According to Mital et al. (2008), the product life-cycle is the time period between the time of product design (concept, plan, design, idea, notion, or thought) and when its production is no longer profitable. So, this is a paradigm that can exploit the aspects related to product management, including those related to the methodologies used in the process of product development. In the context of the "factory as a product" concept, the life-cycle paradigm can be properly extended to be applied in the factory development.

The life-cycle paradigm helps interpret the specific stages that occur throughout product management. Likewise, this can be providential for the determination of the principles and methods used in the development of the future factories.

It can be observed in the literature that the product life-cycle paradigm may be considered from various perspectives: marketing (Kotter, 1992), product management (Saaksvuori \& Immonen, 2005), manufacturing (Kimura, 2005), and computer systems/data management (CIMdata).

Thus, the principles and methods applied in product development, especially the determination of the life-cycle phases, should be adopted in factory development projects under the concept of "factory to the product". 


\section{FACTORY, PRODUCT AND PROCESS LIFE-CYCLE}

\subsection{Factory Planning}

The challenge of designing factories is a complex task because there is an interdependence between factory planning, process planning and product development. This task involves a large number of functions and stakeholders, as well as time constraints, and this situation impels a new area of research so that it is possible to develop a virtual factory framework that will meet the challenges of integration and flexibility on the factory design.

Every factory and production facility is a distinctive entity. Each factory is different in terms of its products, processes, layout, structure, human resources and corporate philosophy. However, the common requirement for its survival is the ability that the production facilities have to adapt. Adaptability is becoming a top priority for modern enterprises and is a continuous task for management. Modern factories have to be modular, scalable, flexible, open, agile, and knowledge-based in order to be able to adapt, in real time, to the continuously changing market demands, technology options and government regulations.

One of the factors that have a profound impact in adaptability is flexibility, hence the constant need to strengthen and improve flexibility in the design of production processes and factory planning. However, the flexibility level depends on the particular context of production and market environment. Thus, the need for new methodologies that support the design of the factory as a product is increasingly obvious. But since this is a highly complex task, it is necessary to study and analyze the approaches followed by experts and experienced implementers in the field.

A first notion that must be present at this stage is the fact that the design of the factory cannot be separated from the planning process and product design, particularly due the functional interdependencies and due to the similarity and parallelism observed in their life-cycle, as can be seen in Fig. 1.

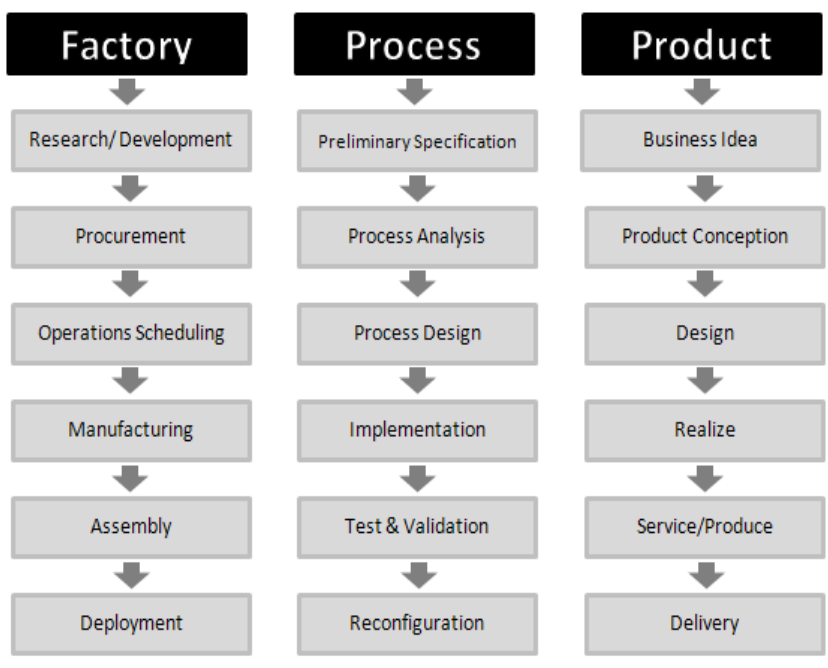

Fig. 1. Factory Planning
In fact, with the introduction of the formal interdependency concept described previously, a factory planning conception can be carried out using knowledge on the life-cycle paradigm, the process planning, factory planning and new product development (NPD).

The new improvements in manufacturing engineering focus on the optimization and value creation for products, processes and technologies over their entire life-cycle (Westkämper, 2006).

Given that the research methodology and knowledge in the product life-cycle approach are more mature, it is advantageous to use this approach to deal with all the factory and process life-cycle.

The Product Life-Cycle (PLC) approach is essential to achieve sustainability by expanding the focus from the production site to the entire factory. By approaching all these entities according to the life-cycle paradigm and by implementing a life-cycle management capability in a factory, considerable benefits are achieved (Westkämper, 2006): Reducing resource use and improving the technical performance, in various stages of the factory's and product's life, shorter time to market, lower costs and reducing rework.

A sustainable factory life-cycle picks up the paradigm of Factories as a Product in order to harmonize and fully integrate the Factory Life-Cycle Phases with the phases of the product life-cycle. The synchronization of these two LifeCycles can be achieved with the approach of Concurrent Engineering. Within this approach, the different phases of the factory life-cycle are mapped to the corresponding phases of the product life-cycle. Furthermore, synergetic effects between Product-Planning and Investment Planning, for example, are pushed by a partitioning strategically planning and technological development. Figure 2 shows the synchronization of the sustainable product and factory lifecycle (Westkämper, 2006).

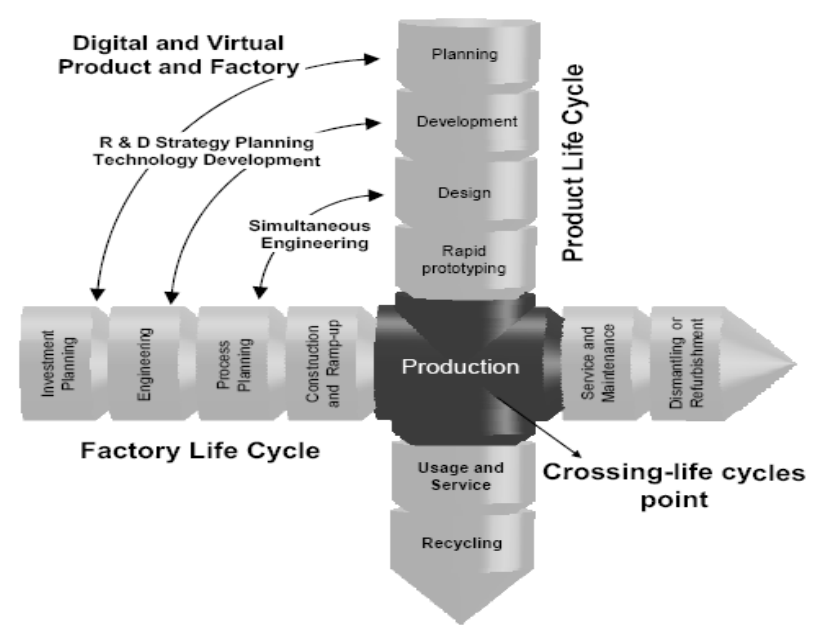

Fig. 2. Factory Life-Cycle vs Product Life-Cycle

Based on this, an integrated Life-Cycle Management for the factory is able to react more efficiently to occurring changes and turbulences in the factory environment. 


\subsection{Factory Optimization}

The Virtual Factory, deployed according to the VFF concept, has to be permanently synchronized with the Real Factory aiming to achieve time and cost savings in the design, rampup, management, evaluation and reconfiguration of the Real Production itself. The Real Factory, interacting in terms of feedbacks and of data required to set-up and update the simulation system, closes the loop.

One of the most relevant phases of the Factory Life-Cycle occurs during the Operation and Maintenance phase. Throughout this phase, the Virtual Factory Framework is fundamental in the improvement and optimization of factory. In order to accomplish this task, a Factory Image is necessary to establish a connection with the physical factory by means of data collection (both in terms of sampling strategies and mechanisms to access the field and of data feeding to simulation), also considering critical hardware in the control loop situations.

Within the identified architecture, the Factory Image will be detailed and developed inside the VFF project in order to provide data for simulations aimed at:

- factory planning, design and reconfiguration;

- $\quad$ production line configuration and balancing;

- $\quad$ production scheduling/planning;

- verification and commissioning of automation software on real factory;

- production maintenance (error/fault detection, diagnosis and recovery).

Among the topics that the VFF will address in order to sustain continuous factory optimization are:

- New, holistic, and robust metrics for the measurement and comparison of factory performance with Key Performance Indicators;

- Optimized allocation of operations or maintenance personnel to the factory workstations;

- Methods to gathering either factory starts or preventive maintenance events;

- Improved methods for the estimation of workstation or factory capacity;

- Decision Support systems to aid decision making in production planning and scheduling;

- "What if" analysis to evaluate production scenarios;

- Reactive support to deal with disruptive events;

- A process-step-centric perspective of factories as opposed to the conventional workstation view presented in the traditional way.

\subsection{Key Benefits}

The implementation of a holistic, modular, open and scalable Virtual Factory is meant to achieve clear, well-identified and measurable goals for real production systems. This will provide greater effectiveness to the next generation factory, particularly in terms of the improvement of product quality and increased productivity of the processes that make it possible to reduce ramp-up and product delivery time, as well as production costs, thus bringing higher competitiveness. Some of the benefits expected are the following.

Product and Process Life-cycle integration: In order to set up a foundation for factory planning, it is necessary to establish the phases that can be standardized in the context of factory design. The intention is to harmonize the life-cycle of the product and the factory and so convergence makes it possible to use simulations in the design of an object-oriented collaborative product from the point of view of the virtual factory to the real factory.

Re-use of useful Knowledge and Good Practices: Knowledge is embedded and carried through multiple entities. The development of methods and techniques to formalize the knowledge is becoming more and more important because a company can improve its performance if it is capable of generating, absorbing, capturing and sharing intellectual assets, knowledge and good practices. This could help increase productivity, reduce processing times and re-work and improve product quality, and also optimize the exploitation of industrial resources, improve the communication among different teams and promote innovation inside the company

Real-time Decision Support: One of the critical challenges of the decision support in general is how quickly we can make good decisions. This will be important to increase the confidence of workers and managers to make decisions because they can predict and anticipate the problems arising from choices made. Moreover, improving performance often requires assimilating, analyzing, and tracking the metrics that show how a company is performing against its business strategic and operational goals. With VFF we see a fundamental shift in the operations of businesses when they combine strategic data with operational data. This shift can be achieved through the materialization of the concept around interaction between a virtual factory and real factory environment. The decision maker in the scope of VFF framework will have the opportunity to evaluate the proposed alternatives in a shorter time, and they will have more confidence in the agreed solution. For example, manufacturing companies could use simulation tools to evaluate the process of re-configuring not only to the products and its variants but also to the factory facilities in order to reach higher flexibility.

Reduction of global response time: VFF is expected to improve the factories' flexibility and adaptability to the market needs thanks to higher effectiveness and efficiency in the design, re-configuration and re-engineering of the factory resources and facilities. The framework also aims at supporting and enabling companies to achieve the ability to 
perform an efficient ramp-up management against the background of Time-To-Market, Time-To-Volume and a strict management of cost, quality and product complexity.

Cost savings: The effective virtual representations of the entire production systems, fostering multi-loop evaluation procedures, multi-dimensional target systems, knowledgedriven decision support and faster problem-solving subprocesses, makes it possible to save costs and time in the evaluation of existing and designed processes, thus resulting in a radically enhanced production quality.

Overall Continuous Improvement: In order to achieve performance outcomes, some inputs may be taken in account, such as, design, operation, maintenance and Improvement practices that should be identified and related with critical Key Performance Indicators. The KPI results would make it possible not only to analyze and evaluate the processes and methods, but also to enable the desired harmonization and performance comparison between the Virtual Factory and the Real Factory, which would be very useful for factory planning. This way, it is possible to achieve a reliable comparison between the two worlds in order to match their performance ("as-is" and "to-be" performances).

\section{VIRTUAL FACTORY FRAMEWORK}

The Virtual Factory develops designs of manufactured components (geometric models), as well the tools and facilities in order to make it possible to define, model and implement any manufacturing process. Moreover, the designs can be updated when corrections or improvements occur in the virtual factory, allowing the process plans to be downloaded into the real factory and thus used for production. So, the envisaged innovative framework (Virtual Factory Framework) leads to major time and costs savings while simulating a dynamic complex behavior over the entire life-cycle factory.

Another very important aspect today is the need to increase the level of flexibility and agility to quickly respond to the market. This requires an improvement in the level of productivity and quality through process improvement and integration of the various activities of the factory, as well as their interactions with external agents. So, there must be a continual quest to improve the design of the factory and its facilities in order to reduce the time to ramp up production and product delivery. In fact, in the context of the virtual factory, we can deal with problem solving in highly complex tasks and in the increasing demand for adaptability, cost, efficiency, durability, reliability, scalability and security (Pedrazzoli et al., 2007).

The challenge is to design the factory of the future considering the synchronization of the planning process of the virtual factory with the real factory. This is achieved through component-based simulation software in order to improve the factory process planning and optimize complex dynamic factory behavior, once viewed as a complex longlived product.

\subsection{VFF Project Aims}

The ongoing European project "Holistic, extensible, scalable and standard Virtual Factory Framework (VFF)" within FP7 aims to foster and strengthen the primacy of Future European Manufacturing by defining the next generation VFFs. The desired outcomes are major time and cost savings for manufacturing companies, while increasing performance in the design, management, evaluation and reconfiguration of new or existing facilities. This project researches and applies models and ideas underlying the creation of the foundations of a new conceptual framework to implement the next generation of Virtual Factory. At the same time, the aim is to lay the foundations for future applications in this research area.

The reference model is based on two key concepts such as the "factory as a product" and "non-linear, non-deterministic planning methodology", represented in Fig. 3 (bellow), which basically consists of the following four pillars:

Reference Model provides a coherent pattern extensible Data Model Factory that considers the holistic needs of modeling and scalable data management and production engineering running in real time.

$V F$ Manager defines and determines the features of abstract objects that represent the factory, its processes and equipment, orchestrating this according to the factory data model defined in Pillar I.

Functional Modules envisage the implementation of different methods, tools and services for the factory design, reconfiguration, management, operating on the same Common Space of Factory Abstract Object, established in Pillar II.

Knowledge Repository and Good Practice is seen as the engine of the concept VFF to model various complex systems so that it is possible to properly understand the methods adapted.

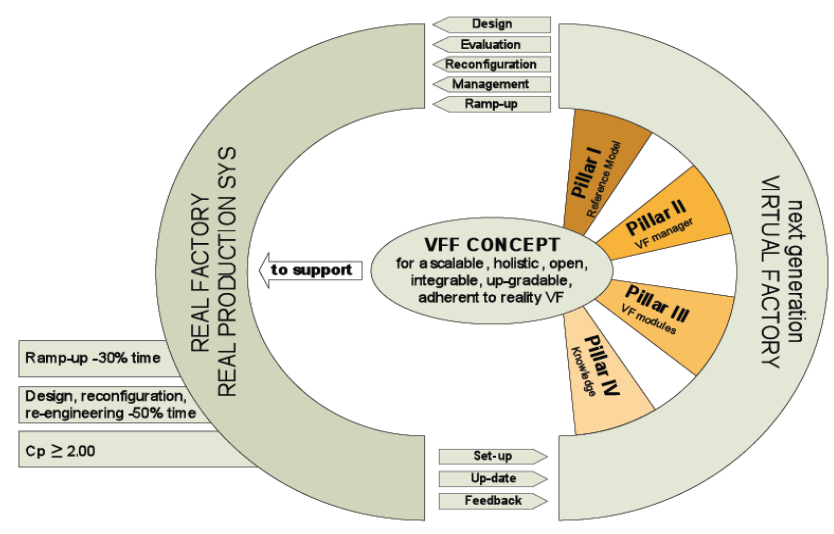

Fig. 3. VFF project concept

The desirable data synchronization and orchestration between the Virtual Factory and the Real Factory (monitoring the KPIs, simulation, design project, system control, and so on), impose the fundamental challenge that is to reach an adequate 
communication between the VF manager and the VF Modules, as well as interaction with humans and ICT.

A data repository takes also plays an important role in this framework because it supports the continuous improvement and design of the products and factory process.

This project aims to set measurable goals for the real production system, such as the following measures: time for factory planning, reconfiguration, reengineering $-50 \%$, the rump-up time of $30 \%$, the capability index $\mathrm{Cp}>=2.0$. It also includes the democratization of knowledge as an important qualitative indicator.

This work is part of an initial analysis performed by the paper's authors. It addresses the specification phases of the VFF project design. Currently, ongoing tasks tackle the requirement analysis of the Virtual Factory Framework. In parallel, various industrial scenarios have been developed in order to evaluate and validate the framework construction.

The development activities are running in specific scenarios, namely: Design \& Optimization, Ramp-up \& Monitoring, Reconfiguration \& Logistics and Next Factory. Almost thirty European partners are involved with the development activities of this project, including research institutes and universities, industries and service companies. The relevant industrial partners committed to the project are: Volkswagen Autoeuropa, Alenia, Audi Hungaria, Compa, Ficep, Frigoglass, Homag and Comau.

In order to strongly enhance perception of the factory as a product notion, factory templates will be developed in this task. The templates will consider design and operation practices throughout the entire life-cycle of the factory. During the creation of the templates, significant efforts will be put in the introduction, development and integration of life-cycle related environmental aspects.

More specifically, such advances include the development of metrics for energy efficiency along with the facilitation of reconfigurability assessments to assist in minimizing waste and increasing resource life-cycle. Thus, the factory templates will also address disassembly and recycling of the plant and its resources, by providing paradigms compatible with modern environmental policies.

\subsection{Factory Template}

Factories should be seen as enormous and complex products that require exhaustive planning processes during the entire life-cycle, from its ideation, to its dismantling.

The factory planning process includes several concepts and elements present in almost all phases of the factory life-cycle. These concepts point to the development of methodologies necessary to plan and execute all engineering tasks associated to successful production and operations.

The Factory Template (FT) can be viewed (Fig. 4) as a metareference model that embodies the VFF factory concept.

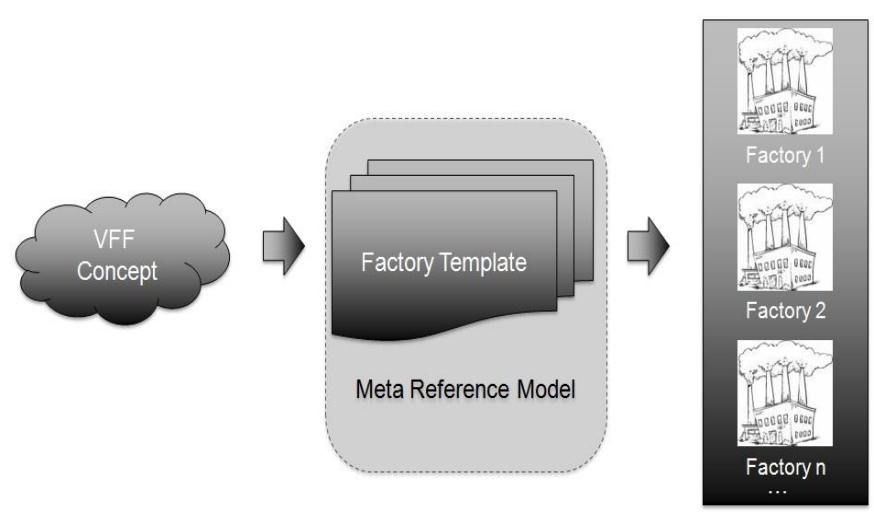

Fig.4. VFF meta-reference model

In a succinct way, Factory Templates can be compared with software patterns with its advantages and final purposes. From software engineering, software patterns are designed patterns fit to provide reusable solutions to a commonly occurring problem. So, as in software science, where the use of templates makes it possible to reduce the development time and to increase programming agility, in the manufacturing management science, it is crucial to use models and standards which allow faster and efficient systems development. These systems present characteristics such as modularity and standardization of industrial processes. Therefore, it is crucial to explore the advantages occurring from the similarity between the Factory Life-cycle Model and Pattern Life-cycle Model (Azevedo \& Almeida, 2010).

In order to strongly enhance the perception of the factory as a product, Factory Templates have been developed in the VFF project. In fact, these templates will: support the operation and evaluation of all factory design phases; show how processes operate; compile perspectives of all the stakeholders involved; structure the information that must be shared and how it should be meaningfully analyzed and reported. Moreover FT will support decision makers in the planning and design of factory life-cycles. So, in order to achieve this purpose, Factory Templates will be divided into two main modeling components: Static and Dynamic strands.

In the first one, factory templates should structure and manage documents, best practices, methods, techniques, processes and knowledge, as well as constraints, goals, requirements and concurrent engineer processes. On the other hand, the dynamic strand will incorporate forecast exercises, internal and external disturbances analysis and performance evaluation of the real factory in comparison with the virtual factory instances.

In fact, all this should be done taking into account different factors and issues, such as production facilities, human roles, information and control systems, energy efficiency, environmental and social issues, among others.

This modeling and standardization concerns are well depicted in the FT. In addition, these templates are capable of facilitating communication aspects among the different manufacturing actors and of contributing to solve problems between people from different departments and origins. 
Another expected benefit occurs when Factory Templates are capable of providing higher performance through the ability to detect malfunctions in the systems before they affect the processes, thus preventing lower performance, increasing costs and higher rework.

Also, it will contribute to develop, in each factory, a multidisciplinary knowledge repository to support the continuous improvement of the factory design throughout each of the Factory Life-Cycle projects.

The role of Knowledge Repository Systems (KRSs) is to facilitate the exchange of knowledge among those who have the knowledge and those who have interested in their application in the manufacturing environment.

\section{CONCLUSION AND FURTHER WORKS}

This work intends to present an innovative approach in the scope of next generation factories development. This work is being done in the context of a multidisciplinary and collaborative European project "Holistic, extensible, scalable and standard Virtual Factory Framework (VFF)" within FP7. This paper addresses mainly the concepts and paradigms that base this innovative approach, namely the "Factory as Product" and Life-Cycle Paradigm, and Factory Templates.

The challenge presented in this ongoing work is to design the factory of the future, taking into consideration the synchronization of the virtual factory's planning process with the real factory.

The accomplished work developed until now has enabled to the project consortium to establish the basic guidelines for the Virtual Factory Framework model, the industrial scenarios validation for proof of principle and prototyping validation, and the definition of the Factory Templates.

Therefore, any future work foreseen in this project involves the development of the reference model for factory and process planning, and the development and implementation of the Virtual Factory Manager, as well as each of the Functional Modules. After these tasks, there should be an integration between the Virtual Factory with and the real factory for final validation.

\section{REFERENCES}

Azevedo A., A. Almeida (2010). The development of factory templates for the integrated virtual factory framework. Unpublished paper accepted to the 20th International Conference in Flexible Automation and Intelligent Manufacturing (FAIM'10).

Mital, A., A. Desai, A. Subramanian and A. Mital (2008). Product Development: A structured approach to consumer product development, design and manufacture. Elsevier, Oxford.

Kotler, P. (2001). Principles of marketing. Prentice Hall, Harlow.

Saaksvuori, A and A. Immonen (2005). Product lifecycle management. 2. ed. Springer, Berlin.
Kimura, F. Engineering Information Infrastructure for Product Lifecycle Management. In: Knowledge and skill chains in engineering and manufacturing. Springer, New York.

CIMdata. Product Lifecycle Management (PLM) Definition. www.cimdata.com/plm/definition.html 05/11/2009.

Pedrazzoli, P., J. Bathelt, G. Chryssolouris, D. Rovere, M. Pappas, C.R. Boër, C. Constantinescu, P. Dépincé, E. Westkämper (2007). High Value Adding VR Tools for Networked Customer-Driven Factory". Proceedings of the 4th International Conference on Digital Enterprise Technology (DET'07).

Westkämper, E., C. Constantinescu and V. Hummel (2006). The migration of life cycle paradigm into the manufacturing engineering. Proceedings of 13th CIRP International Conference. 